\title{
Evaluación clínica de proyectos de inversión y su participación en las ganancias empresariales de los trabajadores
}

José Luis Infante

Facultad de Ingeniería, Universidad Nacional de La Plata. jinfante@ing.unlp.edu.ar

\section{Resumen}

Las evaluaciones clínicas de proyectos de inversión constituyen una forma distinta y novedosa para analizar proyectos que complementa - mas no sustituye - la forma tradicional de evaluación basada en el cálculo de indicadores de riesgo; su aplicación evidencia comportamientos sistémicos que permiten identificar funcionalidades que normalmente no se observan en indicadores sintéticos de eficiencia. Dicha aplicación se ejemplifica en los casos actuales y controvertidos de participación en las ganancias; de lo anterior se derivan recomendaciones clínicas para su utilización en proyectos de inversión.

Palabras clave: proyectos, inversión, clínica, ganancias.

Clasificación JEL: L21, O22 


\title{
Application of investments clinical evaluation to workers' profit sharing
}

\begin{abstract}
Clinical evaluation of investment projects is a different and innovative way for analysis of projects which complements, but does not replace the traditional form of assessment based on the calculation of risk indicators. Its implementation shows evidence of systemic behaviors which permit identification of features which are not normally seen in synthetic efficiency indicators. Implementation in current and controversial cases of profit-sharing is exemplified and from this we derive clinical recommendations for their use in investment projects.
\end{abstract}

Keywords: projects, investment, clinic, profits.

JEL Classification: L21, O22

\section{Introducción}

Este trabajo tiene como objetivo central introducir una forma complementaria y adicional para la evaluación de proyectos de inversión sobre la base de análisis clínicos. A su vez, y como objetivo secundario, se pretende evidenciar sus competencias utilizando como ejemplo la conveniencia o no de la participación en ganancias empresariales a los trabajadores.

El ámbito académico donde se desarrolla puede ser el mundo de la economía, en particular, la microeconomía aplicada; también el de la administración, específicamente, la preparación y evaluación de proyectos. Establecer esa discusión no es objeto de este artículo, pues no se profundizará en tal disyuntiva, sino que en un trabajo posterior se establecerán las diferenciaciones del caso.

Es importante precisar el problema que se trata, pues en las formas tradicionales de análisis normalmente encausan la discusión formalizando el problema y probando resultados sobre la base de un conjunto de indicadores que pretenden sintetizar la información y explicar resultados. Un mayor detalle de lo expuesto indica que el modelador procede a estimar un flujo de caja del proyecto que se trata y sobre él aplica indicadores económicos y otros seleccionados de un tablero de evaluación, como puede ser el valor actual neto, el valor económico agregado, la tasa interna de retorno y otros tantos conocidos. Como es bien sabido, proceder de tal manera 
implica formular económicamente un proyecto para los efectos de su evaluación. Es por ello que a este tipo de trabajos se les conoce con el nombre genérico de evaluación de proyectos de inversión. El conocimiento agregado en este tipo de trabajos y las normas higiénicas de los procedimientos a modo de buenas prácticas de manufactura (BPM) (Krajewski, 2002) se encuentran altamente desarrollados en muchos trabajos generalistas como Infante (2001), Park (2009), Sapag Chaín (2007), Sullivan (2004), entre otros.

Para el caso de clínicas de proyectos, no existe bibliografía específica ni se encuentra tan desarrollada; sin embargo, las funcionalidades clínicas en economía y empresas no son nuevas, pues existen antecedentes desarrollados por Saporosi (1997) donde se utiliza la frase clínica empresaria para destacar análisis administrativos que permitan encontrar causas sobre diagnósticos a efectos observables o esperables; por su parte, Sachs (2005) utiliza la frase clínica económica para describir formas posibles de análisis de causas en problemas de pobreza; finalmente, y más recientemente, Churba (2010) utiliza el término business therapy para describir métodos recomendables para la búsqueda de soluciones a conflictos empresariales.

En definitiva, un proceso de preparación y evaluación de proyectos implica la aceptación o no de un plan productivo diseñado por medio de las técnicas de la producción, de las administraciones específicas (recursos humanos, financiera, etc.) y de la comercialización por la aplicación de indicadores.

Una clara diferencia implica establecer una evaluación clínica. En estos casos no se evalúa un indicador, sino en qué medida puede ser informativo. El estudio clínico analizará comportamientos endógenos, pero fundamentalmente sobre la base de la racionalidad de las estimaciones ejercidas. Para mayor claridad considérese lo siguiente: si un proyecto asume costos por $\mathrm{C}$ e ingresos por I, será rentable en la medida que I $>$ C. Si en dicho proyecto su tasa interna de retorno supera la medida de corte $^{1}$ será además viable. Hasta ese punto informaría una evaluación tradicional de proyectos; sin embargo, una clínica se preguntará, por ejemplo, y entre otras cosas, en qué medida $\mathrm{C}$ y/o I reflejan el estado más creíble de C e I. ${ }^{2}$ Con lo

\footnotetext{
${ }^{1}$ Tasa de atracción o de costo de oportunidad.

${ }^{2}$ Obsérvese que se ha utilizado la palabra creíble y no probable, ya que la medida de la probabilidad puede tener el mismo problema o enfermedad endógena que sus estimaciones.
} 
expuesto se planteará la forma de evaluación clínica de proyectos y se aplicará al caso de participación de los trabajadores en las ganancias.

\section{Ruta del trabajo}

En este trabajo se presenta una descripción de las razones por las cuales se opta por el término clínico para estos análisis. Luego se presenta el caso de estudio que comienza con una exposición de lo que se entiende por participación en ganancias, particularizando después un caso de aplicación. Con ello se procede con la evaluación bajo formas tradicionales y se ensaya un tipo de análisis clínico sobre la base de un modelo microeconómico. Para mayor claridad, se concluye el trabajo con un ejemplo numérico. Finalmente, se presentan las conclusiones.

\section{Evaluación clínica de proyectos}

Fundamentos básicos de clínicas de proyectos. Analogías intuitivas con las clínicas médicas.

Primero se fundamentará el uso de la palabra clínica como adjetivo del sustantivo evaluación. Etimológicamente, clínica hace referencia a un lugar donde hay camas, pues proviene del griego kliné 'cama', que deriva del verbo klinéin 'inclinarse'; estos datos e información más precisa pueden encontrarse en Soca (2009). Por su parte, según la Real Academia Española, clínica proviene del latín clinice y éste del griego $\kappa \lambda \imath v \imath \kappa \eta$, de $\kappa \lambda \imath v \eta$, que significa lecho. De todos sus posibles significados interesa aquel que indica que clínica refiere a un conjunto de las manifestaciones de una enfermedad. Quizás sea esta definición aquella que más precisamente permite identificar la razón de su inclusión para diferenciar una forma de evaluar proyectos.

Haciendo una analogía con la ciencia de la salud, si un proyecto tiene problemas para alcanzar el estado de ganancias es porque algo que debía ser no ha sido; es como si estuviera enfermo. En los seres vivos, bacterias, virus, sobre esfuerzos, problemas genéticos ocasionan que una persona no pueda realizar las actividades que pretende realizar a condición de un resultado esperado. Nada diferente sucede con un proyecto. Una fantasía en su diseño sería similar a un problema genético, quizás un tumor maligno. Pérdidas de información competitiva, mala calidad en la ejecución de los trabajos, problemas políticos empresariales, nuevas tecnologías o 
falta de financiamiento aparecen como bacterias o virus, dependiendo de su magnitud o visibilidad, y en todos estos casos sería oportuno establecer en qué medida el proyecto es resistente o no vulnerable a estos acosos para así determinar si conviene o no diseñar mecanismos de $\operatorname{control}^{3}$ que inhiban en la medida de lo posible las interferencias o, ya establecidas, minimizar el daño.

Tales clasificaciones en relación con qué tipo de analogía corresponde a algún efecto visible en los proyectos sería razón de un trabajo específico donde pueda sustanciarse los argumentos con ejemplos u otras formas. El presente trabajo, introductorio y aplicado, sólo se limita a describir causas y efectos posibles en un tipo de análisis clínico en un caso conflictivo como es la participación o no de los trabajadores en las ganancias empresariales.

Volviendo a la comparación, en la naturaleza de la palabra clínica se encuentra una relación que se establece entre una persona con conocimiento que indaga y observa los efectos para comprender qué sucede. Esta analogía, como se explicó, puede aplicarse a la preparación y evaluación de proyectos de inversión. Es evidente que no existe en este caso la queja por dolores o fiebre, sino que, quizás peor aún, debe ser descubierta.

En términos genéricos, así como existe una clínica preventiva en seres vivos, podrán ejercitarse estudios ex ante $e^{4}$ en los proyectos de inversión que faciliten descubrir y resolver a bajo costo posibles fuentes de improductividades. Así como existen clínicas cuando el paciente enfermo consulta qué le sucede, existirán estudios ex post que buscarán razones para producir las correcciones que permitan, por lo menos, beneficios en el plazo temporal restante. ${ }^{5}$ En esta instancia surge como natural inquietud preguntarse ¿en qué difiere entonces la práctica de evaluación de un proyecto respecto al análisis clínico de proyectos?

\footnotetext{
${ }^{3}$ Por ejemplo, y a modo intuitivo, mecanismos de control preventivos funcionarían como vacunas, mientras que controles por excepción funcionarían como antibióticos.

${ }^{4} \mathrm{La}$ palabra ex ante refiere a preinversión. La palabra ex post se utiliza para los casos durante y posterior a la inversión.

${ }^{5}$ Adviértase que un estudio ex post, que cuenta con información de la ejecución del proyecto, induce alternativas de mejoras ex ante para los tiempos que siguen.
} 


\section{Diferencias entre evaluaciones tradicionales y análisis clínicos}

La formulación y evaluación de proyectos tradicional permite dimensionar el flujo de ingresos y egresos futuros del proyecto de inversión y el cálculo de indicadores de récord para efectuar una toma de decisión. En los casos de proyectos privados, la eficiencia que pretenderá medir los indicadores de récord será fundamentalmente de rentabilidad. ${ }^{6}$ Luego, si dicha dimensión cumple con la producción de las necesidades por satisfacer y los indicadores permiten observar que tales asignaciones son económicamente viables, no existe ninguna otra etapa posterior de estudio más allá de variabilidades o riesgo.

La clínica, a diferencia de la formulación y evaluación tradicional, indaga adicionalmente sobre las relaciones funcionales en los sistemas internos ${ }^{7}$ que pudieran derivar en improductividades, canibalización en los estudios de demanda, costos de agencia sobredimensionados o que pueden ser susceptibles de quedar temporalmente sobredimensionados, capacidad informativa de los datos o calidad de éstos, entre otras situaciones.

Desde este enfoque, a efectos de generalizar, se podría suponer que los estudios de formalización y evaluación de proyectos tradicional forman parte de la clínica de proyectos de inversión. Una clínica básica y elemental sólo indica una esperanza de rentabilidad a constancia de los estados funcionales de las estimaciones. ${ }^{8}$ Una clínica más avanzada, la cual es objeto de este trabajo, observará las relaciones en los efectos económicos que resultan del dimensionado previsto. Con ello, de observarse malos acondicionados ${ }^{9} \mathrm{u}$ otras deficiencias, el técnico actuante se encargará de hacerlo saber para efectuar las correcciones que correspondan al problema observado.

\footnotetext{
${ }^{6}$ Véanse Chiavenato (2005), Hitt (2006) e Infante (2001).

${ }^{7}$ Más precisamente subsistemas internos.

${ }^{8}$ Obsérvese que no se habla de constancia de estimaciones, pues la evaluación de proyectos estudia riesgos, pero definidos sobre un valor base de la variable de estudio; es decir, su estado y un entorno de variación de esos estados centrado en la estimación antedicha. Por ejemplo, si se evalúa una estimación de ingresos de 1000000 y una variabilidad del 10\%, los estados posibles serán escogidos de un entorno de 900000 y 1100000 . Una clínica sobre ingresos discutirá las relaciones funcionales que indican que la variable sea estimada en 1000000 y su variabilidad esperada. Claro que para ello un primer estado de análisis indica llegar a medir el valor de 1000000.

${ }^{9}$ Término técnico que, intuitivamente, hace referencia a problemas informativos o asignaciones de oportunidad ineficientes.
} 


\section{Ejemplo de aplicación de análisis clínicos a la conveniencia de utilizar forma- tos de participación en ganancias en los operarios asignados a un proyecto}

\section{Aclaraciones preliminares}

El problema que se pretende resolver racionalmente implica decidir si en la distribución de ganancias de un proyecto de inversión se asigna o no parte de estas ganancias a los trabajadores. Antes de avanzar con el análisis se hace la salvedad de que lo que se pretende analizar es si la medida — es decir, la participación en ganancias- induce incentivos en el juego económico, en especial, en el jugador empresario. En ningún momento este artículo avanza sobre una forma particular de implementación, sino más bien considera formas genéricas. Como ya se mencionó, es interés de este trabajo aplicar análisis clínicos para comprender si existen o no incentivos para reconocer la participación en ganancias para los trabajadores, más allá que las normas de gobierno lo exijan o no. Obsérvese que si hubiera incentivos, los trabajadores y empresarios reconocerían en el proyecto un juego no cooperativo donde la participación en las ganancias formaría parte. ${ }^{10}$ Ello no es menor, puesto que la existencia de incentivos en equilibrios $\mathrm{Nash}^{11}$ disminuyen los costos de administración de gobierno. Entonces, la cuestión que se intentará responder es, más allá de la normativa vigente, si le conviene a los empresarios distribuir ganancias.

\section{Descripción de la participación en ganancias por utilizar}

La participación en las ganancias ${ }^{12}$ consiste en una entrega de dinero que hace la empresa a sus trabajadores financiando tal acto por medio del fondo de utilidades distribuidas. Se establece un porcentaje que es afectado de dicho origen y distribuido en relación con el nivel salarial de los trabajadores. Los aportes normalmente no son bonificables ni remunerativos. ${ }^{13}$

Su materialización puede incluir o no auditorías sindicales sobre los informes contables para determinar si el monto de utilidad distribuido es el correcto. Ya sea en

\footnotetext{
${ }^{10}$ Respecto a juegos cooperativos y no cooperativos podrá consultarse Gibbons (1993).

${ }^{11}$ Véanse Gibbons (1993) y Porto (2002 y 2004).

${ }^{12}$ Véase De Castro (1974).

${ }^{13}$ Es decir, no se le aplican porcentajes por antigüedad u otro mérito y no debe utilizarse para determinar montos jubilatorios o indemnizaciones.
} 
este u otro caso, sólo se diferencia costos de administración sin que sea relevante para decidir o no conveniencias. Para discriminar claramente cómo informa una evaluación tradicional respecto de un enfoque clínico, primero se ensayará una respuesta desde la evaluación de proyectos para luego proceder con las diferencias que destaca el tipo de análisis que se propone.

\section{Evaluación tradicional}

Supóngase un nivel de producción “q”, un precio de mercado "p", un mercado competitivo imperfecto con rendimientos marginales decrecientes tal que $p=p(q)$ con $\partial \mathrm{p} / \partial \mathrm{q}<0 .{ }^{14}$ Considérese que los costos vienen dados por la función $\mathrm{c}=\mathrm{c}(\mathrm{q})$ y existe un capital hundido F. Obsérvese a su vez un caso típico de producción de bienes, por tanto, $\partial c / \partial q>0$ atento a que los rendimientos serán decrecientes. ${ }^{15}$ Suponiendo que el inversor adopta el indicador tasa interna de retorno (TIR) como índice sintético, el cálculo que refiere la evaluación del proyecto será $\sum_{\mathrm{j}} \mathrm{S}_{\mathrm{j}} /(1+\mathrm{TIR})^{\mathrm{j}}=0$ siendo variado $\mathrm{j}=0 . . n$ y $\mathrm{S}_{\mathrm{j}}=[\mathrm{pq}-\mathrm{c}(\mathrm{q})-\mathrm{F}]_{\mathrm{j}}$. El proyecto será rentable, bajo condición necesaria y suficiente, si $\sum_{j} S_{j}>0$, lo que brindará TIR $>0$. Véase que el proyecto en sí produce ese nivel de rentabilidad independientemente de quién se apropie de ella. Ahora, si la medida de TIR es asignada a los inversores y a los trabajadores, suponiendo $\mathrm{i}_{\mathrm{T}}$ la parte de TIR asignada a los inversores e $\mathrm{i}_{\mathrm{T}}$ la parte de TIR asignada a los trabajadores, la denominada participación en ganancias, a condición que $i_{1}>0$ y $i_{1}>$ TIR con l=I,T, el inversor elegirá o no hacer la inversión en la medida que $i_{1}>r$, donde $r$ es la tasa de costo de oportunidad.

Luego, si $i_{1}<$ TIR siempre y en todos los casos existirán más posibilidades que el inversor elija proceder con la inversión si no hay participación en las ganancias, ya que en ese caso $i_{1}=$ TIR. Por ello, será racional que el inversor siempre prefiera que $\mathrm{i}_{\mathrm{T}}=0$. Se destaca entonces que adoptando decisiones en el marco expuesto, las preferencias racionales del inversor invocan que no haya participación en ganancias, ya que ello puede influir en mermas de inversiones.

Cabe destacar que se ha utilizado el indicador TIR, pero no cambiarían los resultados de utilizar otra medida de rentabilidad. Es sabido que la TIR cuenta con francas ventajas relativas a otros indicadores debido a su capacidad de arbitraje, a su

\footnotetext{
${ }^{14}$ Por competencia imperfecta.

${ }^{15}$ Las conclusiones que se alcanzan bajo esta restricción no se oponen si fuera el caso de rendimientos marginales crecientes.
} 
medida que refiere a porcentajes, a ser un indicador absolutamente endógeno, muy utilizado y conocido, y uno de los pocos que permite comparaciones casi directas entre inversiones financieras e inversiones productivas; sin embargo, también presenta desventajas, fundamentalmente en la relevante suposición de la reinversión de utilidades en proyectos de igual rentabilidad, multiplicidad de resultados ante flujos no simples y otras cuestiones que le quitan atractivo, pero académico. Dado que tanto las ventajas como las desventajas no influyen en los objetivos de este trabajo se les considerará relajadas, es decir, no oponibles.

\section{Evaluación bajo análisis clínico}

Considérese que el directorio de una empresa piensa en coparticipar parte de sus ganancias que se obtengan en el proyecto de inversión antes descrito apropiando una cantidad " $x$ " tal que $0<x<1$. Su problema será elegir convenientemente dicha cuantía de " $x$ " para realizar su ofrecimiento al sindicato o grupo de trabajadores, el cual persigue consensuar con la organización laboral para que se materialicen incentivos en los trabajadores con el fin de que sean posibles disminuciones secuenciales de costos de producción con el mismo nivel de calidad. Esos resultados podrán ser alcanzados por una disminución de accidentes y enfermedades, mayores tiempos productivos, menores caídas tecnológicas, menores pérdidas por negligencia, descuidos y hasta colusiones; en definitiva, todas fuentes de disminución de improductividades. De ser esto posible, los costos reflejarán la función de costos conocida menos la disminución de la improductividad la que, en función de los razonables incentivos de los trabajadores, será mayor cuanta mayor participación en las ganancias se les otorgue. Dicha disminución operará sobre el costo de la siguiente unidad que se produzca toda vez que debe existir una instancia informativa donde se evidencien los menores costos. La medida del costo marginal, $\partial \mathrm{c} / \partial \mathrm{q}$, al ser positiva por rendimientos marginales decrecientes, y considerando que la improductividad mejorada suma un valor " $\mathrm{k}$ " tal que $0<\mathrm{k}<1$, el costo de producción podrá ser modelado por la expresión $\mathrm{c}(\mathrm{q}, \mathrm{x})=\mathrm{c}(\mathrm{q})-(1-\mathrm{x}) \mathrm{k} \partial \mathrm{c} / \partial \mathrm{q}$. Posteriormente, al existir un instrumento de control y medición protocolizado "I" por el cual el directorio y el sindicato confían que refleja convenientemente las ganancias alcanzadas, la determinación de "x" —cuestión pasiva para los trabajadores toda vez que, en primer juego ${ }^{16}$, dicha medida la dispone para ser ofertada por el directorio- resulta de un proceso de racionalización:

$\operatorname{MAX}_{(\mathrm{q}, \mathrm{I})}[\mathrm{p}(\mathrm{q}) \mathrm{q}-\mathrm{c}(\mathrm{q}, \mathrm{x})] \mathrm{x}=\operatorname{MAX}_{(\mathrm{q}, \mathrm{I})}[\mathrm{p}(\mathrm{q}) \mathrm{q}-(\mathrm{c}(\mathrm{q})-(1-\mathrm{x}) \mathrm{k} \partial \mathrm{c} / \partial \mathrm{q})] \mathrm{x}$

${ }^{16} \mathrm{El}$ primer juego indica la oferta que la empresa haría al sindicato para suscribir el convenio de participación. 
Recordando el punto antes tratado sobre el directorio inversor que elije de acuerdo con el indicador TIR y éste resulta de $\sum_{\mathrm{j}} \mathrm{S}_{\mathrm{j}} /(1+\mathrm{TIR})^{\mathrm{j}}=0$ siendo variado $\mathrm{j}=0 . . \mathrm{n}$ y $\mathrm{S}_{\mathrm{j}}=[\mathrm{pq}-\mathrm{c}(\mathrm{q})-\mathrm{F}]_{\mathrm{j}}$, un proceso de racionalización implicará elegir el nivel de producción "q" y luego seleccionar el valor de " $\mathrm{x}$ " que cumpla con $\operatorname{MAX}_{\mathrm{q}}(\mathrm{TIR})=$ $\operatorname{MAX}_{q}\left[\sum_{j} S_{j} /(1+T I R)^{j}=0\right]^{17}$ con $j=0 . . n$ y $S_{j}=[p q-c(q)-F]_{j}$, puesto que TIR es una medida endógena en la maximización planteada. Por su parte, en atención a que $\operatorname{MAX}_{\mathrm{q}}\left[\sum_{\mathrm{j}} \mathrm{S}_{\mathrm{j}} /(1+\mathrm{TIR})^{\mathrm{j}}=0\right]$ puede reescribirse sin perder generalidad como $\sum_{\mathrm{j}}$ $\operatorname{MAX}_{\mathrm{q}}\left(\mathrm{S}_{\mathrm{j}}\right) /(1+\mathrm{TIR})^{\mathrm{j}}=0$ el problema por resolver será $\operatorname{MAX}_{\mathrm{q}}\left(\mathrm{S}_{\mathrm{j}}\right)$, donde $\mathrm{S}_{\mathrm{j}}=[\mathrm{pq}-$ $\mathrm{c}(\mathrm{q})-\mathrm{F}]_{\mathrm{j}} \mathrm{y} F$ son invariantes tanto a " $\mathrm{j}$ " como a "q" por definición de capital hundido resulta que $S_{j}=[p q-c(q)]_{j}$; reescribiendo la expresión de $c(q)$ en función de " $x$ " $y$ " $k$ " se encuentra el mecanismo de selección de "q" propuesto $\operatorname{MAX}_{(\mathrm{q}, \mathrm{I})}[\mathrm{p}(\mathrm{q}) \mathrm{q}$ $\mathrm{c}(\mathrm{q}, \mathrm{x})] \mathrm{x}=\operatorname{MAX}_{(\mathrm{q}, \mathrm{I})}[\mathrm{p}(\mathrm{q}) \mathrm{q}-(\mathrm{c}(\mathrm{q})-(1-\mathrm{x}) \mathrm{k} \partial \mathrm{c} / \partial \mathrm{q})] \mathrm{x}$.

Para maximizar se elige el "q" que determina el punto crítico de la expresión. En consecuencia se diferencia y se iguala a 0 encontrándose $\partial \mathrm{p} / \partial \mathrm{q} \mathrm{qx}+\mathrm{px}-\partial \mathrm{c} / \partial \mathrm{q} \mathrm{x}+$ $\mathrm{kx} \partial 2 \mathrm{c} / \partial \mathrm{q} 2-\mathrm{kx} 2 \partial 2 \mathrm{c} / \partial \mathrm{q} 2=0$.

Excluyendo el caso $\mathrm{x}=0$, ya que ello inviabiliza desde un enfoque económico el proyecto de inversión, se puede simplificar $\mathrm{x}$, con lo que $\partial \mathrm{p} / \partial \mathrm{q} q+\mathrm{p}-\partial \mathrm{c} / \partial \mathrm{q}+\mathrm{k}$ $\partial 2 \mathrm{c} / \partial \mathrm{q} 2-\mathrm{kx} \partial 2 \mathrm{c} / \partial \mathrm{q} 2=0$; despejando $\mathrm{x}$ permite alcanzar la medida $\mathrm{x}=(\partial \mathrm{p} / \partial \mathrm{q} \mathrm{q}+$ $\mathrm{p}-\partial \mathrm{c} / \partial \mathrm{q}+\mathrm{k} \partial 2 \mathrm{c} / \partial \mathrm{q} 2) /(\mathrm{k} \partial 2 \mathrm{c} / \partial \mathrm{q} 2)$, la cual queda garantizado que existe, puesto que se puso como condición que $0<\mathrm{k}<1$ y que los rendimientos marginales son decrecientes. ${ }^{18}$

Tomando como condición de participación en las ganancias que $0<x<1$, si $x>0$ resulta que

$(\partial \mathrm{p} / \partial \mathrm{q} \mathrm{q}+\mathrm{p}-\partial \mathrm{c} / \partial \mathrm{q}+\mathrm{k} \partial 2 \mathrm{c} / \partial \mathrm{q} 2) /(\mathrm{k} \partial 2 \mathrm{c} / \partial \mathrm{q} 2)>0$

$\partial \mathrm{p} / \partial \mathrm{q} q+\mathrm{p}>\partial \mathrm{c} / \partial \mathrm{q}-\mathrm{k} \partial 2 \mathrm{c} / \partial \mathrm{q} 2$

$\partial \mathrm{p} / \partial \mathrm{q} \mathrm{qp} / \mathrm{p}+\mathrm{p}>\partial \mathrm{c} / \partial \mathrm{q}-\mathrm{k} \partial 2 \mathrm{c} / \partial \mathrm{q} 2$

у $\eta=(\partial \mathrm{q} / \partial \mathrm{p})(\mathrm{p} / \mathrm{q})$ la medida de la elasticidad, al reagrupar resulta que $\mathrm{p}>(\partial \mathrm{c} / \partial \mathrm{q}-\mathrm{k}$ $\partial 2 \mathrm{c} / \partial \mathrm{q} 2) /(1+1 / \eta)$.

\footnotetext{
${ }^{17} \mathrm{~A}$ los efectos de no distraer el objetivo de este trabajo en otras cuestiones que requerirán ampliaciones del presente, se supondrá que el flujo del proyecto es simple y que es factible la reinversión de utilidades en proyectos de igual o superior TIR.

${ }^{18} \mathrm{Bajo}$ rendimientos marginales decrecientes $\partial 2 \mathrm{c} / \partial \mathrm{q} 2>0$, mientras que para rendimientos constantes $\partial 2 \mathrm{c} / \partial \mathrm{q} 2=0$ cuestión que no es factible en producciones reales. El caso de rendimientos marginales crecientes debería ser estudiado bajo un modelo diferente al planteado.
} 
Tomando el otro extremo, si $\mathrm{x}<1$ sucede que $\mathrm{p}<(\partial \mathrm{c} / \partial \mathrm{q}) /(1+1 / \eta)$ resultando finalmente que

$$
(\partial \mathrm{c} / \partial \mathrm{q}-\mathrm{k} \partial 2 \mathrm{c} / \partial \mathrm{q} 2) /(1+1 / \eta)<\mathrm{p}<(\partial \mathrm{c} / \partial \mathrm{q}) /(1+1 / \eta)
$$

Es decir, dependiendo de la elasticidad, existe una gama de precios de mercado donde es eficiente y racional la participación en las ganancias. Esta conclusión resulta seriamente dañada cuando el mercado se transforma en rígido, cuestión donde el daño se expande también a los consumidores.

Como puede observarse, la clínica desafía abiertamente las conclusiones de la evaluación de proyectos tradicional indicando marcos de comportamiento económicos donde puede suceder que acuerdos no cooperativos y fuera de escenarios de "mesa despareja" 19 permitan maximizar ganancias con inclusión voluntaria de participación de ganancias.

\section{Ejemplo numérico de aplicación}

A continuación se ensayará un ejemplo numérico para dar mayor claridad a la propuesta metodológica presentada.

En función de evitar abundar en información que no aporta nada sustancial sobre la temática planteada, sólo se considerará las cuantías y valoraciones objeto de análisis relajando y, en consecuencia, parametrizando otras estimaciones necesarias.

Supóngase que el proyecto tratado en el modelo microeconómico cuenta con el detalle de inversiones, ventas y costos que se observa en el cuadro 1. Manteniendo la nomenclatura ya utilizada, la misma se ingresará en la fila siguiente a la del enunciado de las variables. Ello ayudará a la identificación de los términos teóricos discutidos.

\footnotetext{
${ }^{19} \mathrm{Si}$ el acuerdo es no cooperativo, las partes han encontrado un equilibrio donde ambas maximizan sus posiciones estratégicas. Que sea fuera de efectos de "mesa despareja" indica que las partes cuentan con poder de decisión nivelados. Para una mayor aclaración confróntese Kritek (1998) e Infante (2001).
} 
Cuadro de evaluación bajo análisis tradicional

El cuadro 1 detalla los resultados que propone una evaluación de proyectos tradicional antes de análisis de riesgos.

\section{Cuadro 1}

Resultados sin análisis clínicos

\begin{tabular}{|c|c|c|c|c|c|c|c|}
\hline Año & Inversión & Precio & Costo & Cantidades & $\begin{array}{c}\text { Flujo neto } \\
\text { antes de la } \\
\text { participación }\end{array}$ & Participación & $\begin{array}{c}\text { Flujo neto } \\
\text { después de la } \\
\text { participación }\end{array}$ \\
\hline & $\mathrm{F}$ & $\mathrm{p}$ & $c(q)$ & $q$ & $p q-c(q)-F$ & $\% \mathrm{P}$ & $\mathrm{pq}-\mathrm{c}(\mathrm{q})-\mathrm{F}-\% \mathrm{P}$ \\
\hline 0 & 1000000 & & & & -1000000 & & -1000000 \\
\hline 1 & & 10.0 & 6.0 & 100000 & 400000 & 40000 & 360000 \\
\hline 2 & & 10.0 & 6.0 & 100000 & 400000 & 40000 & 360000 \\
\hline 3 & & 10.0 & 6.0 & 100000 & 400000 & 40000 & 360000 \\
\hline 4 & & 10.0 & 6.0 & 100000 & 400000 & 40000 & 360000 \\
\hline \multirow[t]{3}{*}{5} & & 10.0 & 6.0 & 100000 & 400000 & 40000 & 360000 \\
\hline & \multicolumn{4}{|c|}{ VAN $(0 \%)$} & 1000000 & 100000 & 800000 \\
\hline & \multicolumn{4}{|c|}{ TIR } & $28.65 \%$ & & $23.44 \%$ \\
\hline
\end{tabular}

El proyecto supone una inversión (indicada como F) de \$1 000000 que retorna en un plazo de cinco años para la producción y venta mayorista de productos elásticos. Las estimaciones suponen un precio unitario sugerido competitivo de $\$ 10$ y costos unitarios por \$6. Éstos resultan a partir de las esperanzas matemáticas de las variables que los explican. Las cantidades por producir suponen el uso del $90 \%$ de la capacidad instalada a un turno de labor.

Dicha estimación permite adoptar una política conservadora que permite asumir sin inconvenientes las demandas del mercado y las necesidades de mantenimiento. Los costos también adoptan las necesidades adicionales de personal por vacaciones, siniestros por seguridad e higiene, capacitaciones y las necesidades de insumos adicionales por caídas tecnológicas e improductividades por mal uso y deficiencias de métodos y controles. Los costos de administración se encuentran incluidos a excepción de impuestos directos. 
Se observa que aceptando una participación en ganancias del 10\% la TIR disminuye sensiblemente de $28.65 \%$ a $23.44 \%$ después de dichas distribuciones. $\mathrm{La}$ recomendación racional sería entonces propiciar medidas políticas que eviten participar ganancias.

\section{Cuadro de evaluación bajo análisis clínico}

El análisis microeconómico, expresado en el apartado Descripción de la participación en ganancias por utilizar, indica que la participación en ganancias genera incentivos. Para observar tal efecto se estudian las fuentes de los incentivos tanto en los trabajadores como en las empresa; he allí la función clínica. Se observa que los trabajadores, en presencia de un método protocolizado de cuantificación periódica de ganancias, tienen suficientes incentivos intuitivos para cuidar insumos, evitar caídas tecnológicas y estar más atentos a la eventual presencia de accidentes o enfermedades. Recuérdese que la capacitación y, fundamentalmente, la atención del trabajador son cruciales a la hora de disminuir este tipo de siniestros. A partir de antecedentes de otras firmas y de las superintendencias de gobierno, se puede construir una curva de aprendizaje del trabajador que le permite suponer eficiencias en costos debido a las razones mencionadas, entre otras.

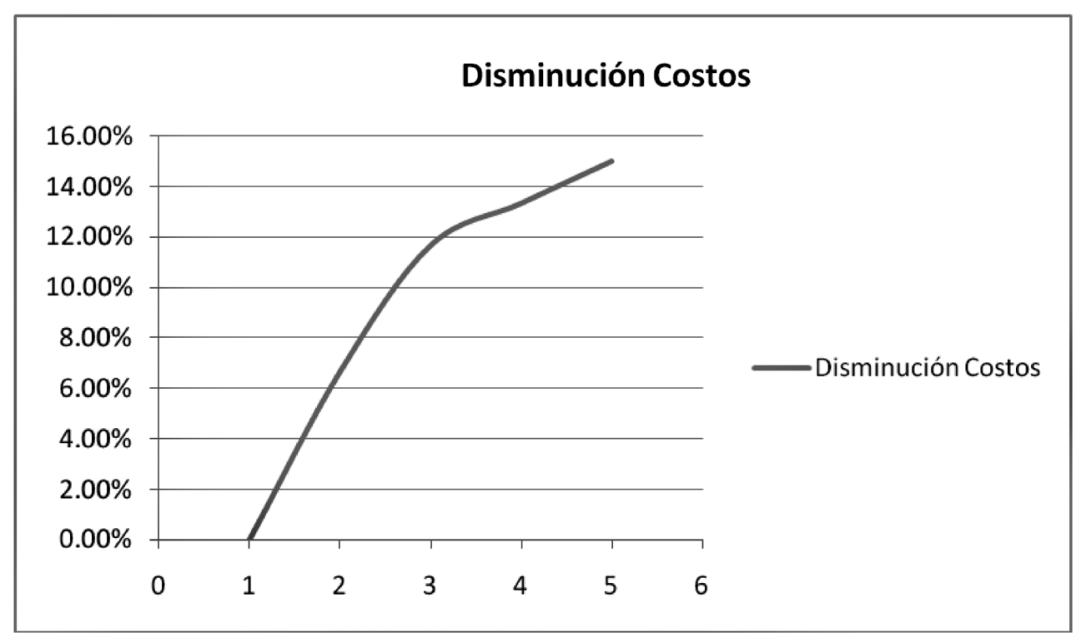


La curva presentada resulta del siguiente cuadro de eficiencias ${ }^{20}$

\begin{tabular}{c|c}
\hline Año & $\begin{array}{c}\text { Disminución } \\
\text { de costos }\end{array}$ \\
\hline 1 & $0.00 \%$ \\
\hline 2 & $6.67 \%$ \\
\hline 3 & $11.67 \%$ \\
\hline 4 & $13.33 \%$ \\
\hline 5 & $15.00 \%$ \\
\hline
\end{tabular}

Nótese que las eficiencias se alcanzan en el tiempo una vez que los trabajadores en promedio, o si se quiere el trabajador representativo, comprenden la importancia de sus propios controles materializándose en mayores ingresos para él y su entorno. Esa comprensión proviene de entender y aprender mejores técnicas, así como creer que su esfuerzo vale al recibir los pagos incrementales de la empresa. De ello resulta un sensible cambio en el flujo del proyecto que se presenta a continuación.

\section{Cuadro 2}

\section{Resultados con análisis clínico}

\begin{tabular}{|c|c|c|c|c|c|c|c|}
\hline Año & Inversión & Precio & Costo & Cantidades & $\begin{array}{c}\text { Flujo neto } \\
\text { antes de la } \\
\text { participación } \\
\end{array}$ & Participación & $\begin{array}{l}\text { Flujo neto después } \\
\text { de la participación }\end{array}$ \\
\hline & $F$ & $p$ & $c(q)$ & $q$ & $p q-c(q)-F$ & $\begin{array}{c}{[p(q) q} \\
-(c(q)-(1- \\
x) k \partial c / \partial q)] \\
(1-x)-F\end{array}$ & $\begin{array}{c}{[p(q) q-(c(q)} \\
-(1-x) k \partial c / \partial q)] x-F\end{array}$ \\
\hline 0 & 1000000 & & & & -1000000 & & -1000000 \\
\hline 1 & & 10.0 & 6.0 & 100000 & 400000 & 40000 & 360000 \\
\hline 2 & & 10.0 & 5.6 & 100000 & 440000 & 44000 & 396000 \\
\hline 3 & & 10.0 & 5.3 & 100000 & 470000 & 47000 & 423000 \\
\hline 4 & & 10.0 & 5.2 & 100000 & 480000 & 48000 & 432000 \\
\hline 5 & & 10.0 & 5.1 & 100000 & 490000 & 49000 & 441000 \\
\hline & \multicolumn{4}{|c|}{ VAN $(0 \%)$} & 1280000 & 128000 & 1052000 \\
\hline & \multicolumn{4}{|c|}{ TIR } & $34.06 \%$ & & $28.68 \%$ \\
\hline
\end{tabular}

${ }^{20}$ Los valores suman eficiencias por siniestros y por disminuciones de caídas tecnológicas en industrias continuas. De requerirse ampliaciones de estos conceptos se recomienda revisar el trabajo de Krajewski (2002). 
Claramente se evidencia que la aplicación de la participación en ganancias es buena para todos, pues la TIR del proyecto después de distribuir ganancias es superior, aunque humildemente, toda vez que $28.65 \%$ es inferior a $28.68 \%$. Ello interesa a las partes involucradas y al estado, quien se apropiará de un mayor volumen de impuestos, dado que el proyecto en sí ofrece un retorno del 34.06\%. La empresa alcanza mejor resultado, ya que $28.68 \%$ de TIR es superior al $23.44 \%$ y en general se ganará en eficiencia toda vez que el escenario que se presenta, pues $28.65 \%$, rentabilidad del proyecto antes de distribuir ganancias, es inferior a $28.68 \%$, medida de rentabilidad después de distribución. En otras palabras, podrá alcanzarse menores costos de control administrativo, puesto que los incentivos juegan a favor de la distribución. Por otra parte, el proyecto dirigido a la empresa es más atractivo, puesto que $28.68 \%$ de TIR es superior a $23.44 \%$ y contará con mayores posibilidades de ejecución toda vez que supera un nivel superior de tasa de corte. Finalmente, los trabajadores alcanzan un mayor volumen de beneficios ya que una distribución de $\$ 128000$ supera una distribución de $\$ 100000$, lo que implica entonces mayores incentivos a la aplicación cierta de la norma.

\section{Efectos del análisis clínico en el ejemplo expuesto}

El análisis clínico ex ante, como se observa en este ejemplo, y siguiendo la visión de Sachs y de Saporosi ya comentada, permite identificar senderos o caminos donde se puede maximizar la eficiencia productiva del proyecto. Colateralmente, permite identificar la necesidad de precisar información o mejorar la calidad de datos, identificar restricciones administrativas, etc. Claro es que de ninguna manera cambia un proyecto. Eso corresponde a reingenierías y otras aplicaciones.

La evaluación tradicional, al trabajar sobre estimaciones de alta probabilidad, no permite identificar comportamientos o fuentes de mejoras. Ello sólo se alcanza con el análisis de las clínicas sobre el proyecto. Como se observa en el ejemplo, los valores de las estimaciones sobre la base de supuestos promedios no facilitan observar eficiencias porque éstas requieren aplicar otros modelos de comportamientos que deben ser validados por conocimientos del medio. Quizás vale entonces preguntar por qué no incluir desde un inicio las ventajas de las mermas en costos en la evaluación tradicional, ya que, en definitiva, los indicadores, en este caso la TIR, resulta de una misma forma de cálculo. La respuesta es que no es objetivo de una evaluación tradicional tal cosa toda vez que incluye valores promedios con inclusión de estimaciones de costos que contemplen comportamientos promedios del mercado. Recuérdese en este punto que la pregunta que se pretende contestar 
es qué sucede si algo se hace distinto a cómo se está haciendo; es decir, si se paga ganancias cuando en realidad no se está pagando o se está pagando sólo en el mercado formal o se está pagando bajo una formalidad diferente. Como el escenario es nuevo, los datos existentes no lo reflejan; por lo tanto, tampoco lo hacen las estimaciones que existen, por lo que se requiere en consecuencia otro análisis: el clínico. Otra función clínica, no presentada en este trabajo, discute la calidad informativa del dato, malos acondicionados y otros aspectos. Por último, cabe señalar que el ejemplo no evalúa el efecto del cambio en la elasticidad, el cual puede ser una posible fuente de una ampliación de este trabajo.

\section{Conclusiones}

En el presente trabajo se han discutido las diferencias que existen entre análisis de proyectos de inversión tradicional y análisis clínico. De igual forma, se ha precisado el porqué del término clínico en proyectos de inversión, reconociéndose precedentes de uso de este término para cuestiones empresariales generales y fenómenos económicos de distribución de riqueza y pobreza.

Por otra parte, se ha indicado que los mecanismos normales de preparación y evaluación de proyectos no permiten identificar en todos los casos, y con un mayor grado de claridad, los efectos esperables de la interrelación de los procesos generadores de costos e ingresos, sus incentivos y efectos sistémicos, mientras que los análisis clínicos sí evidencian las tendencias en las funcionalidades de dichos costos e ingresos pudiendo obtener un mayor conocimiento sobre resultados esperables como razón adicional que permita juzgar la capacidad de los informes de rentabilidad. En consecuencia, la ventaja de su uso complementa los mecanismos conocidos.

Para clarificar la discusión normativa expresada, se ejemplifican las diferencias en la evaluación de la conveniencia de la participación en las ganancias y bajo qué esquemas de procedimientos puede esperarse mayor eficiencia por aplicación de dicho formato. Se detalla que el interés de este trabajo se centra en la relevancia del estudio clínico para observar si la participación en ganancias para los trabajadores produce o no incentivos más allá de formas de aplicación o normativas vigentes.

Finalmente, se procedió con un encuadre microeconómico con el fin de evidenciar si las empresas podrían tener incentivos para ofertar porcentajes de participación 
en las ganancias encontrándose que existen medidas que serían eficientes subyaciendo información reconocida como válida por sindicatos o agrupación de trabajadores y productos de sustitución elástica.

\section{Referencias}

Chiavenato, I. (2005). Introducción a la teoría general de la administración. México: McGraw Hill.

Churba, A. (2010). Business therapy, el método para liderar hacia mejores resultados. España: Aguilar.

De Castro, C.B. (1974). Cogestión y participación en las ganancias. Buenos Aires: eudeba.

Fontaine, E. (2002). Evaluación social de proyectos. México: Alfaomega.

Gibbons, R. (1993). Un primer curso de teoría de juegos. Barcelona: Antoni Bosch.

Goldratt, E. (2009). La decisión: cómo elegir la mejor opción. Buenos Aires: Granica.

Hitt, M. et al. (2006). Administración. 9a. ed. México: Pearson Educación.

Infante, J.L.(2001). Economía y producción. Buenos Aires: Nueva Librería.

Krajewski, L. et al. (2002). Administración de operaciones. Estrategia y análisis. México: Pearson Educación.

Kritek, P. B. (1998). La negociación en una mesa despareja. Un abordaje práctico para trabajar con las diferencias y la diversidad. Buenos Aires: Granica.

Park, Ch. S. (2009). Fundamentos de ingeniería económica. 2a. ed. México: Pearson Educación.

Porto, A. (2002). Microeconomía y federalismo fiscal. Buenos Aires: Universidad de La Plata. 
(dir.) (2004). Disparidades regionales y federalismo fiscal. Buenos Aires: Universidad de La Plata.

Real Academia Española (2001). Diccionario de la lengua española. 22a. ed. Madrid: Espasa Calpe.

Sachs, J. (2005). The end of poverty. Economic Possibilities for Our Time. Londres: Penguin Press.

Samuelson, P. (2006). Economía. México: McGraw Hill.

Sapag Chaín, N. (2007): Preparación y evaluación de proyectos. México: Pearson Educación.

Saporosi, G. (1997). Clínica empresaria. Buenos Aires: Macchi.

Soca, R. (2009). La fascinante historia de las palabras. Montevideo: Argumento.

Sullivan, W.G. (2004). Ingeniería económica de DeGarmo. 12a. ed., México: Pearson Educación.

$$
\text { (A) }
$$

